\title{
Editorial
}

\section{Smart Cities: Recent Trends, Methodologies, and Applications}

\author{
Damianos Gavalas, ${ }^{1}$ Petros Nicopolitidis, ${ }^{2}$ Achilles Kameas, ${ }^{3}$ Christos Goumopoulos, ${ }^{4}$ \\ Paolo Bellavista, ${ }^{5}$ Lampros Lambrinos, ${ }^{6}$ and Bin $\mathbf{G u o}^{7}$ \\ ${ }^{1}$ Department of Product and Systems Design Engineering, University of the Aegean, Syros, Greece \\ ${ }^{2}$ Department of Informatics, Aristotle University of Thessaloniki, Thessaloniki, Greece \\ ${ }^{3}$ School of Science and Technology, Hellenic Open University, Patras, Greece \\ ${ }^{4}$ Department of Information \& Communication Systems Engineering, University of the Aegean, Samos, Greece \\ ${ }^{5}$ Department of Computer Science and Engineering, University of Bologna, Bologna, Italy \\ ${ }^{6}$ Department of Communication and Internet Studies, Cyprus University of Technology, Limassol, Cyprus \\ ${ }^{7}$ School of Computer Science, Northwestern Polytechnical University, Xian, Shaanxi, China \\ Correspondence should be addressed to Damianos Gavalas; dgavalas@aegean.gr
}

Received 24 September 2017; Accepted 25 September 2017; Published 25 October 2017

Copyright (C) 2017 Damianos Gavalas et al. This is an open access article distributed under the Creative Commons Attribution License, which permits unrestricted use, distribution, and reproduction in any medium, provided the original work is properly cited.

Worldwide forecasts indicate that the size and population of cities will increase further. This immense growth will put a strain on resources and pose a major challenge in many aspects of everyday life in urban areas, such as the quality of services in the medical, educational, environmental, transportation, public safety, and security sectors, indicatively. Thus, novel methods of management must be put in place for these cities to remain sustainable. The wide adoption of pervasive and mobile computing systems gave rise to the term of "smart cities," which implies the ability of sustainable city growth by leading to major improvements in city management and life in the above-mentioned sectors and other aspects such as energy efficiency, traffic congestion, pollution reduction, parking space, and recreation. This has been made possible in recent years due to the widespread availability of commodity low-power sensors, smart phones, tablets, and the necessary wireless networking infrastructure, which, along with technologies such as AI and management of big data, may be utilized to address the challenges of sustainable urban environments.

The motivation behind this special issue has been to solicit cutting-edge research relevant to technologies, methodologies, and applications for smart cities. The special issue has attracted 19 submissions. Following a rigorous review process (including a second review round), 7 outstanding papers (acceptance rate 36.8\%) have been finally selected for inclusion in the special issue. The accepted papers cover a wide range of research subjects in the broader area of smart cities, including service delivery, service recommendation, user privacy, crowdsensing, and vehicular networks.

The paper "Crowdsensing Task Assignment Based on Particle Swarm Optimization in Cognitive Radio Networks" by L. Zhai and $\mathrm{H}$. Wang proposes an optimal algorithm based on particle swarm optimization to solve the problem of assigning wireless spectrum sensing tasks to mobile intelligent terminals in Cognitive Radio Networks. The algorithm employs crowdsensing principles and takes into account several factors including remaining energy, locations, and costs of mobile terminals.

The paper "An ARM-Compliant Architecture for User Privacy in Smart Cities: SMARTIE-Quality by Design in the IoT" by V. Beltran et al. introduces the IoT-Architecture Reference Model (IoT-ARM) and describes its application within the European-funded project, SMARTIE. The paper discusses the architectural aspects of SMARTIE which support efficient and scalable security and user-centric privacy.

The paper "Fault Activity Aware Service Delivery in Wireless Sensor Networks for Smart Cities" by X. Zhang et al. considers the problem of fault-aware multiservice delivery in Wireless Sensor Network environments, wherein the network performs secure routing and rate control in terms of fault activity dynamic metric. The authors propose a distributed 
framework to estimate the fault activity information based on the effects of nondeterministic faulty behaviours and then present a fault activity geographic opportunistic routing (FAGOR) algorithm addressing a wide range of misbehaviours.

The paper "A Hybrid Service Recommendation Prototype Adapted for the UCWW: A Smart-City Orientation" by $\mathrm{H}$. Zhang et al. deals with the problems of cold start and sparsity when considering service recommendation in ubiquitous computing environments. To alleviate these problems, the authors propose a hybrid service recommendation prototype utilizing user and item side information for use in the Ubiquitous Consumer Wireless World (i.e., a novel wireless communication environment that offers a consumercentric and network-independent service operation model, allowing the materialization of a broad range of smart city scenarios).

The paper "Data Dissemination Based on Fuzzy Logic and Network Coding in Vehicular Networks" by X. Tang et al. presents a data dissemination scheme for vehicular networks based on fuzzy logic and network coding. The scheme addresses the problems of high velocity, frequent topology changes, and limited bandwidth, so as to efficiently propagate data in vehicular networks. Fuzzy logic is used to compute the transmission ability for each vehicle while network coding is utilized to reduce transmission overhead and accelerate data retransmission.

The paper "Unchained Cellular Obfuscation Areas for Location Privacy in Continuous Location-Based Service Queries" by J.-N. Luo and M.-H. Yang describes an unchained regional privacy protection method that combines query logs and chained cellular obfuscation areas to ensure location privacy and effectiveness in location-based services (LBS). The proposed method adopts a multiuser anonymizer architecture to prevent attackers from predicting user travel routes by using background information derived from maps (e.g., traffic speed limits).

The paper "A Real-Time Taxicab Recommendation System Using Big Trajectories Data” by P. Chen et al. proposes a novel algorithmic approach for recommending either a vacant or an occupied taxicab in response to a passenger's request. The recommendation algorithm indicates the closest vacant taxicab to passengers; otherwise, it infers destinations of occupied taxicabs by similarity comparison and clustering algorithms and then recommends to passengers an occupied taxicab heading to a nearby destination.

We do hope that this special issue will be of considerable interest to the Wireless Communications and Mobile Computing's audience, highlighting state-of-the-art trends, methodologies, and applications in smart city environments.

\section{Acknowledgments}

We would like to sincerely thank the authors of all the submitted papers for considering our special issue and the Wireless Communications and Mobile Computing as a potential publication venue for their research results. We would also like to especially thank the authors of the accepted papers for their effort in revising and improving their work, occasionally, several times, in response to reviewers' comments. In addition, we would like to thank the anonymous reviewers for doing an excellent job in reviewing the submitted papers and making this special issue possible. Last but not least, we take this opportunity to thank the Editorial Board for giving us the opportunity to organize this special issue, which we sincerely believe provides a fresh, relevant, and useful overview of ongoing research in the multifaceted area of smart cities.

\author{
Damianos Gavalas \\ Petros Nicopolitidis \\ Achilles Kameas \\ Christos Goumopoulos \\ Paolo Bellavista \\ Lampros Lambrinos \\ Bin Guo
}




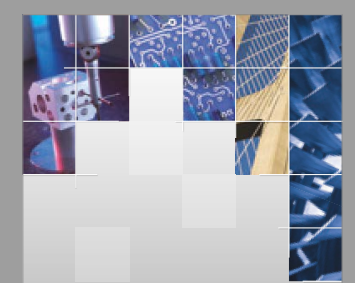

\section{Enfincering}
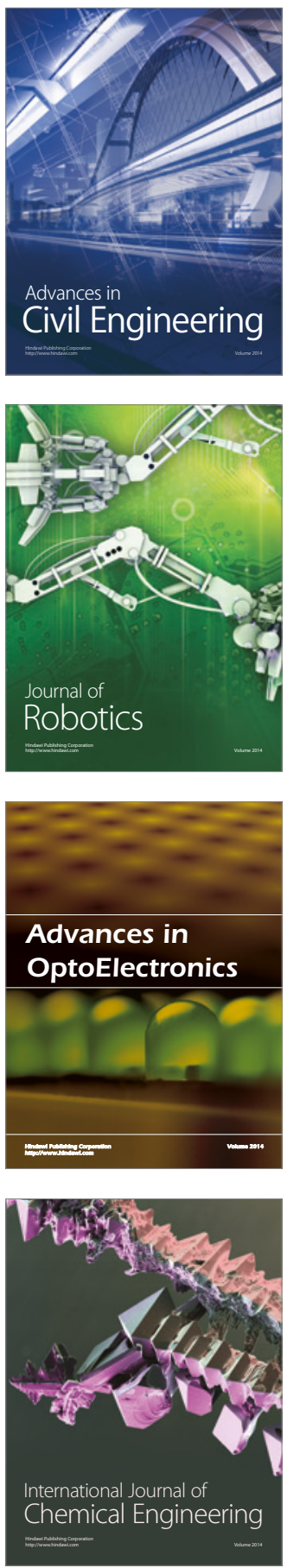

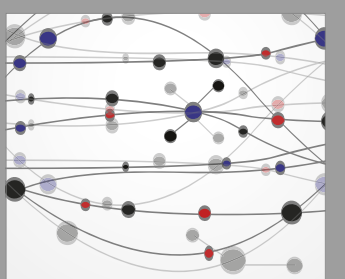

The Scientific World Journal

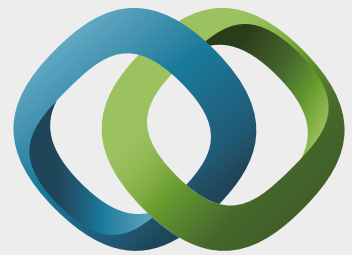

\section{Hindawi}

Submit your manuscripts at

https://www.hindawi.com
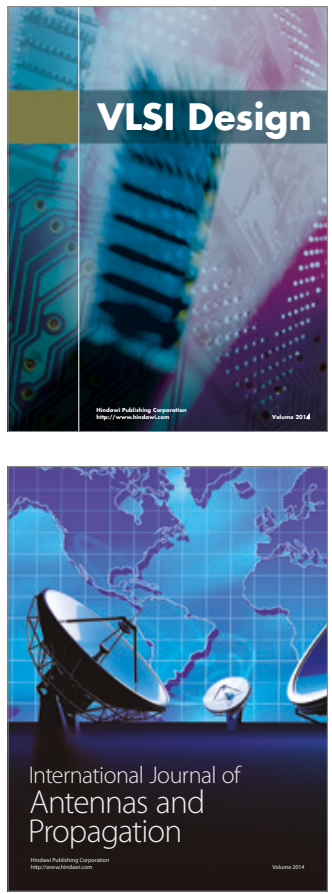

\section{Rotating}

Machinery
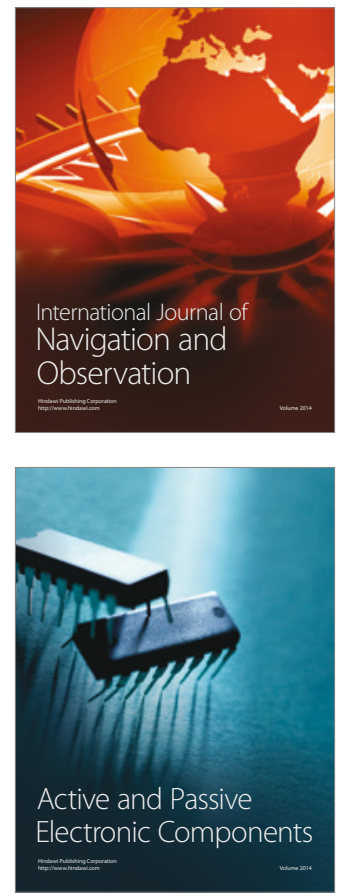
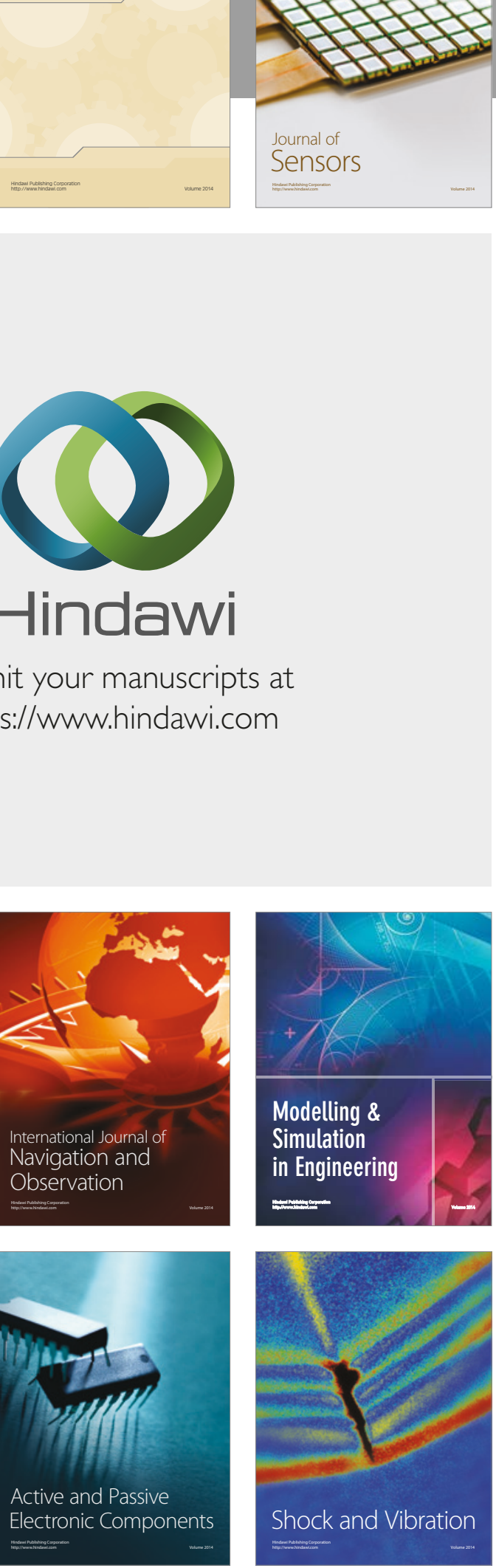
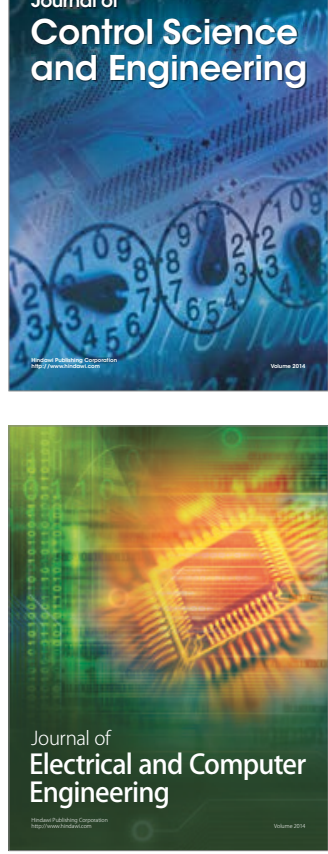

Distributed

Journal of

Control Science

and Engineering
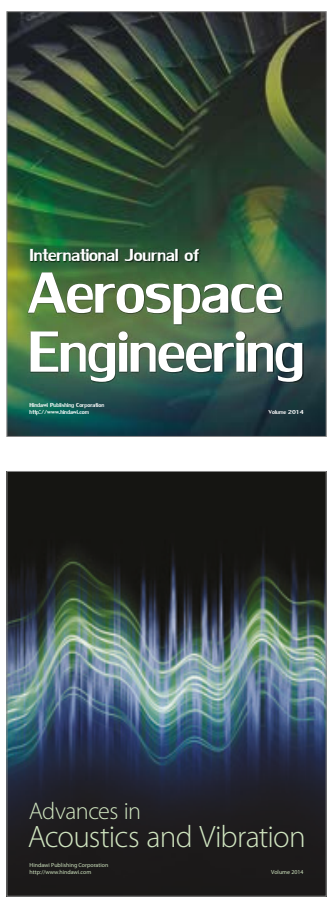

Sensor Networks 